\title{
FIXED POINT THEOREMS FOR PSEUDO MONOTONE MAPPINGS ${ }^{1}$
}

\author{
L. E. WARD, JR.
}

1. Introduction. Recently [7] the author generalized a well-known theorem of Hamilton [1] in the following manner: if $X$ is a continuum each of whose subcontinua is unicoherent and decomposable, then $X$ has the fixed point property for monotone transformations. As a corollary it followed that the same fixed point property obtains for continua each of whose nondegenerate subcontinua has a cutpoint. The argument depended on the order structure of a certain arcwise connected hyperspace of the continuum.

In this note we arrive at the same corollary by a distinctly different and simpler proof. $A u$ fond the argument is essentially the same as one due to Kelley [2] where it was shown that a homeomorphism of a continuum into itself has an invariant, cutpoint-free subcontinuum. (The analogous result for monotone transformations was proved by the author in [6].) The proof of Kelley does not make full use of the properties of homeomorphisms; the essential properties which make his argument work define a class of transformations which we shall term the pseudo monotone mappings.

Finally, we note that our results for pseudo monotone mappings admit a further generalization in the setting of partially ordered topological spaces.

2. Pseudo monotone mappings. Let $X$ and $Y$ be spaces and $f: X \rightarrow Y$ a continuous mapping. We say that $f$ is pseudo monotone if, whenever $A$ and $B$ are closed and connected subsets of $X$ and $Y$, respectively, and $B \subset f(A)$, it follows that some component of $A \cap f^{-1}(B)$ is mapped by $f$ onto $B$. In general this notion is independent of that of a monotone mapping, but in certain applications of interest every monotone mapping is pseudo monotone.

Recall that a continuum (= compact connected Hausdorff space) is hereditarily unicoherent if any two of its subcontinua meet in a connected set.

LEMMA 1. If $X$ is an hereditarily unicoherent continuum and $f: X \rightarrow Y$ is a monotone mapping, then $f$ is pseudo monotone.

Presented to the Society, April 22, 1961; received by the editors December 28, 1960.

1 This research was supported by the United States Air Force through the Air Force Office of Scientific Research of the Air Research and Development Command, under Contract No. AF 49(638)-889. Reproduction in whole or in part is permitted for any purpose of the United States Government. 
Proof. Let $A$ and $B$ be closed and connected subsets of $X$ and $Y$, respectively, such that $B \subset f(A)$. Since $f$ is monotone, $f^{-1}(B)$ is a continuum, and since $X$ is hereditarily unicoherent, $A \cap f^{-1}(B)$ is connected. Hence $f$ is pseudo monotone.

Suppose now that $X$ is a continuum and that $f: X \rightarrow X$ is continuous. A simple maximality argument establishes the existence of a nonempty subcontinuum $Y$, which is minimal with respect to being invariant under $f$. Suppose $Y$ has a cutpoint $p$, with

$$
Y-p=A \cup B
$$

where $A$ and $B$ are disjoint, separated and nonempty. If $f(p)=p$ then the minimality of $Y$ is contradicted, so we may assume $f(p) \in A$ and define $r(Y)=\bar{A}$ by

$$
\begin{array}{ll}
r(x)=x, & x \in \bar{A}, \\
r(x)=p, & x \in \bar{B} .
\end{array}
$$

The mapping $g: \bar{A} \rightarrow \bar{A}$ defined by $g=r f$ is continuous, and the set

$$
K=\bigcap_{n=1}^{\infty}\left\{g^{n}(\bar{A})\right\}
$$

is a subcontinuum of $\bar{A}$ which is invariant under $g$. Thus

$$
f(K) \cap K=r f(K)=g(K)=K
$$

and we infer $K \subset f(K)$. Therefore, if $f$ is pseudo monotone, the set $K \cap f^{-1}(K)$ has a component $K_{1}$ such that $f\left(K_{1}\right)=K$. Inductively we obtain a sequence of subcontinua, $K_{n}$, such that

$$
K_{n} \subset f\left(K_{n}\right)=K_{n-1} \subset \cdots \subset f\left(K_{1}\right)=K .
$$

Clearly, the intersection of this sequence is a nonempty subcontinuum invariant under $f$, and this contradicts the minimality of $Y$. We have proved

Theorem 1. If $X$ is a continuum and $f: X \rightarrow X$ is a pseudo monotone mapping, then $X$ contains a nonempty subcontinuum $Y$ which is minimal with respect to being invariant under $f$. Moreover, $Y$ has no cutpoints.

CoRollary 1.1. If $X$ is a continuum such that each of its nondegenerate subcontinua has a cutpoint, and if $f: X \rightarrow X$ is a pseudo monotone mapping, then there exists $x_{0} \in X$ such that $x_{0}=f\left(x_{0}\right)$.

It has been proved elsewhere [7] that the continua of Corollary 1.1 are hereditarily unicoherent. Therefore, by Lemma 1, we have 
COROLLARY 1.2. If $X$ is a continuum such that each of its nondegenerate subcontinua has a cutpoint, and if $f: X \rightarrow X$ is a monotone mapping, then there exists $x_{0} \in X$ such that $x_{0}=f\left(x_{0}\right)$.

3. A generalization. In [5] the author defined a POTS (= partially ordered topological space) to be a partially ordered set $X$, so topologized that the sets

$$
L(x)=\{a: a \leqq x\}, \quad M(x)=\{a: x \leqq a\}
$$

are closed, for each $x \in X$. Two elements $x$ and $y$ of $X$ are comparable if $x \leqq y$ or $y \leqq x$. In the event $X$ contains a unit, i.e., a unique element $e$ such that $L(e)=X$, we say that the subset $A$ of $X$ is bounded away from $e$ if there exists $y \neq e$ such that $A \subset L(y)$.

The following theorem was proved in [5].

Fixed Point Theorem. Let $X$ be a compact Hausdorff POTS with unit, e. Let $f: X \rightarrow X$ be a continuous, order-preserving mapping satisfying the following conditions.

(i) There exists $x \neq e$ such that $x$ and $f(x)$ are comparable.

(ii) If $x \neq e$ and if $x$ and $f(x)$ are comparable, then either the sequence $f^{n}(x), n=1,2, \cdots$, is bounded away from $e$, or $f^{-1}(x) \cap L(x)$ is nonempty.

Then there exists $x_{0} \neq e$ such that $x_{0}=f\left(x_{0}\right)$.

For the remainder of this paper let us assume that $X$ is a compact Hausdorff POTS with unit $e$, which is endowed with the following two properties.

$(\alpha)$ There exist elements $a, b$ and $p$ of $X$ such that $L(a) \cap L(b)=p$.

( $\beta)$ If $x \in X-L(a) \cup L(b)$ then $p \leqq x$ and each of the sets $L(x) \cap L(a)$ and $L(x) \cap L(b)$ has a supremum.

Let $f: X \rightarrow X$ be continuous and order-preserving, and suppose $f$ maps minimal elements into minimal elements. In addition, suppose $f$ satisfies the following order-theoretic analogue of pseudo monotonicity.

(P) If $x \leqq f(x)$ then $f^{-1}(x) \cap L(x)$ is nonempty.

According to the fixed point theorem above, $f$ has a fixed point distinct from $e$ if $f(x) \leqq x$ for some $x \neq e$. If this does not occur, then by $(\beta)$ and the fact that $f(p)$ is minimal, we have $f(p) \leqq a$ or $f(p) \leqq b$, but not both. Suppose $f(p) \leqq a$; since $f$ is order-preserving, $f(a)$ cannot lie in $L(b)$. Moreover, $f(a)$ cannot lie in $L(a)$ by assumption, so that by $(\beta)$ there must exist

$$
\iota_{1}=\sup (L(f(a)) \cap L(a)),
$$


with $p \leqq t_{1}$. Now $f\left(t_{1}\right) \in X-L(a)$ and, since $p \leqq t_{1}$, it follows that $f(p)$ $\leqq f\left(t_{1}\right)$ and hence $f\left(t_{1}\right) \in X-L(b)$. Applying $(\beta)$ again there exists

$$
t_{2}=\sup \left(L\left(f\left(t_{1}\right)\right) \cap L(a)\right)
$$

with $p \leqq t_{2}$. Because $f$ is order-preserving it follows that $f\left(t_{1}\right) \leqq f(a)$ and hence $t_{2} \leqq f(a)$. Moreover, $t_{2} \leqq a$ so that $t_{2} \leqq t_{1}$. Inductively, we obtain a sequence $t_{n}$ satisfying

$$
t_{n+1}=\sup \left(L\left(f\left(t_{n}\right)\right) \cap L(a)\right), p \leqq t_{n+1} \leqq t_{n} .
$$

Since $t_{n}$ is a decreasing sequence, it must converge to some $t_{0} \leqq t_{n}$. Further, since $t_{n} \leqq f\left(t_{n-1}\right)$, it follows that $t_{0} \leqq f\left(t_{0}\right)$. Condition (i) is now satisfied and (ii) follows from (P) and the above discussion. Hence we infer (compare with a result of A. D. Wallace [4])

Theorem 2. Let $X$ be a nondegenerate compact Hausdorff POTS with unit e, satisfying $(\alpha)$ and $(\beta)$. Let $f: X \rightarrow X$ be a continuous, orderpreserving mapping which maps minimal elements into minimal elements and satisfies $(\mathrm{P})$. Then there exists $x_{0} \in X-e$ such that $f\left(x_{0}\right)=x_{0}$.

It is not difficult to see that Theorem 2 is truly a generalization of Theorem 1. Let $Y$ be a continuum with a cutpoint $p$, and let $f(Y)=Y$ be pseudo monotone. If $X$ is the space of subcontinua of $Y$, endowed with the finite topology [3], and if $f^{*}$ is the mapping of $X$ into itself induced by $f$, then $X$ and $f^{*}$ satisfy the hypotheses of Theorem 2 , where the partial order is taken to be inclusion. Thus $Y$ contains an invariant proper subcontinuum and Theorem 1 follows.

\section{REFERENCES}

1. O. H. Hamilton, Fixed points under transformations of continua which are not connected im kleinen, Trans. Amer. Math. Soc. vol. 44 (1938) pp. 18-24.

2. J. L. Kelley, Fixed sets under homeomorphisms, Duke Math. J. vol. 5 (1939) pp. 535-536.

3. Ernest Michael, Topologies on spaces of subsets, Trans. Amer. Math. Soc. vol. 71 (1951) pp. 152-182.

4. A. D. Wallace, $A$ fixed point theorem, Bull. Amer. Math. Soc. vol. 51 (1945) pp. 413-416.

5. L. E. Ward, Jr., Partially ordered topological spaces, Proc. Amer. Math. Soc. vol. 5 (1954) pp. 144-161.

6. - Continua invariant under monotone transformations, J. London Math. Soc. vol. 31 (1956) pp. 114-119.

7. - A fixed point theorem for monotone mappings, Abstract No. 61T-45, Notices Amer. Math. Soc. vol. 8 (1961) p. 66.

UNIVERSITY OF OREGON 